\title{
Responsive fluorophore aggregation provides spectral contrast for fluorescence lifetime imaging
}

Kelton A. Schleyer ${ }^{+[a, c]}$, Benjamin D. Datko ${ }^{+[a, b]}$, Brandon Burnside ${ }^{[a, b]}$, Chao Cui ${ }^{[a, c]}$, Xiaowei Ma ${ }^{[a, c]}$, John K. Grey ${ }^{[a, b]}$, and Lina Cui*[a,c]

[a] Department of Chemistry and Chemical Biology, UNM Comprehensive Cancer Center, University of New Mexico, Albuquerque, NM 87131, USA

[b] Center for High Technology Materials, University of New Mexico, Albuquerque, NM 87106, USA

[c] Department of Medicinal Chemistry, College of Pharmacy, UF Health Science Center, UF Health

Cancer Center, University of Florida, Gainesville, FL 32610, USA

'These authors contributed equally to the work.

*Corresponding author. Email: linacui@cop.ufl.edu (L. Cui).

\section{Abstract}

Fluorophores experience altered emission lifetimes when incorporated into and liberated from macromolecules or molecular aggregates; this trend suggests the potential for a fluorescent, responsive probe capable of undergoing self-assembly and aggregation and consequently altering the lifetime of its fluorescent moiety to provide contrast between the active and inactive probes. We developed a cyanobenzothioazole-fluorescein conjugate (1), and spectroscopically examined the lifetime changes caused by its reduction-induced aggregation in vitro. A decrease in lifetime was observed for compound $\mathbf{1}$ in a buffered system activated using the biological reducing agent glutathione, suggesting a possible approach for designing responsive self-aggregating lifetime imaging probes. 


\section{Introduction}

The lifetime of a fluorescence event is an intrinsic property of the fluorescent species in question. The same fluorophore can possess unique lifetime values if its population is in two distinct environments, resulting in contrast created by the same reporter ${ }^{1,2}$. Lifetime can be altered chemically, such as by coordination with metal ions $\mathrm{s}^{3,4}$ or through acid-base equilibrium with environmental $\mathrm{pH}^{5,6}$; it is also exquisitely sensitive to small changes in environmental factors like temperature ${ }^{7}$ and solvent viscosity, ${ }^{8,9}$ The lifetime of a fluorophore is influenced by its capacity to undergo non-radiative emission ${ }^{10}$, which is altered by immobilizing factors such as tethering to macromolecules ${ }^{11}$, entrapment in aggregated proteins ${ }^{12}$, or intercalation into $D N A^{13}$. In these examples, the lifetime of the free fluorophore is altered when exposed to a macromolecular aggregate.

Fluorescein isothiocyanate (FITC) has served as a reporter in several lifetime studies involving aggregation or macromolecules. When bound to the active site of the anti-fluorescein antibody Mab 44-20, FITC was observed to have a major lifetime component of $0.37 \mathrm{~ns}$, compared to the 3.86 ns major component observed for the fluorophore free in solution ${ }^{14}$. A poly-L-lysine oligomer bearing multiple FITC pendants was observed to aggregate in solution, giving a FITC lifetime of $0.5 \mathrm{~ns}^{15}$; upon degradation of the oligomer by cathepsins the resulting FITC-lysine monomers reported a restored lifetime of 4 ns.

Aside from FITC, other small molecule fluorophores have reported faster lifetimes when associated with large polymers, aggregates, or biomacromolecules, than when measured as smaller entities $^{11,16,17}$. While the reverse trend has also been reported ${ }^{13,18}$, these findings suggest that fluorescence lifetime is sensitive to local aggregation. From this, we anticipated that incorporating FITC into a self-assembling structure would result in similar lifetime shifts which could serve as a signal for imaging biological events. 
Reporter aggregation is a strategy proposed to improve the efficacy of small molecule imaging of biological events ${ }^{19}$. Aggregation of a non-targeted, small molecule reporter inhibits mobility and promotes retention at the site of activation ${ }^{19-21}$, providing better spatial resolution usually achieved with active-targeting or covalent-labeling imaging strategies. One such aggregation system is built upon the final step in the biosynthesis of luciferin ${ }^{22}$ and involves the condensation of the 1,2-aminothiol group of free cysteine and the nitrile group of 2-cyanobenzothiazole ${ }^{23}$ (CBT). Creating a molecule bearing both functionalities results in a compound that can condense into cyclic oligomers and subsequently selfassemble into nanostructures. This reaction is rapid ${ }^{24}$ (with a second-order rate constant of $9.19 \mathrm{M}^{-1} \mathrm{~s}^{-1}$ ) and can be controlled by caging portions of the 1,2-aminothiol group to create a bio-responsive aggregation system ${ }^{23}$. This system has been used as a platform for a wide variety of imaging methods ${ }^{25-}$ ${ }^{34}$, including steady-state fluorescence imaging ${ }^{20,35-38}$, but it has not been examined as a platform for time-resolved fluorescence imaging.

Considering the inherent sensitivity of fluorescence lifetime to aggregation, this triggered aggregation system may serve as a means of producing contrast in the lifetime of a covalently-linked fluorescent reporter. This scaffold could then be caged by substrates or other moieties to facilitate smart imaging in response to enzymes or cellular conditions of interest, a strategy thoroughly demonstrated by previous work ${ }^{20,25-33,35-38}$. For instance, it is reported that many cancer cell lines produce increased levels of glutathione (GSH) compared to their normal tissue counterparts ${ }^{39-41}$; caging the sulfhydryl group of the scaffold with a reduction-sensitive disulfide bond would facilitate glutathione-responsive lifetime imaging (Figure 1).

\section{Results and Discussion}

Characterization of GSH-triggered aggregation. We designed compound $\mathbf{1}$ by attaching FITC to a simple CBT scaffold (Scheme S1) and tested its response to GSH in PBS buffer. Compound $\mathbf{1}$ as a $100 \mu \mathrm{M}$, 
$10 \mu \mathrm{M}$, or $1 \mu \mathrm{M}$ solution in PBS ( $\mathrm{pH}=7.4$ ) was incubated with $10 \mathrm{mM} \mathrm{GSH}$, and aggregation of the activated (reduced) compound was detected by Nanoparticle Tracking Analysis (NTA). Nanoparticles of 150-200 nm average size were detected when 1 was incubated with GSH (Table S2), similar in size to other iterations of this scaffold ${ }^{25,33,34,37,42}$.

Stead state electronic absorption and emission. To determine the influence of this aggregation on the nature of the attached FITC fluorophore, we examined the steady state electronic absorption, excitation, and emission spectra of 1 before and after activation (Figures 2 and 3). The absorption of the $100 \mu \mathrm{M}$ activated solution of $\mathbf{1}$ (Figure $2 \mathrm{~A}$, black solid) shows two predominate inhomogeneously broaden peaks centered at $2.58 \mathrm{eV}$, and $3.29 \mathrm{eV}$. The absorption of activated $\mathbf{1}$ also shows a steady rise in absorptivity moving toward lower energy caused by scattering (Figure 2A). The broadening of the FITC absorption, $2.58 \mathrm{eV}$, could be resulting from a distribution of polymer sizes upon reaction which then perturbs the FITC moiety in a distribution of arrangements. The reaction, in theory, should not alter the FITC moiety but once the activated product of $\mathbf{1}$ is aggregated many different nano-environments may alter the FITC moiety to a large degree, resulting in broadening. The broadened peak centered at 3.29 $\mathrm{eV}$ is assigned to the product oligomer of $\mathbf{1}$. Evidence for the assignment can be seen in Figure 3 as overlapping excitation spectra from $2.74 \mathrm{eV}(453 \mathrm{~nm}$ ) emission (yellow solid squares) with the absorption. This also gives more evidence for the new emission seen in Figure 2B, at $2.74 \mathrm{eV}$ (453 nm) predominantly coming from the resulting product 1 oligomer. The excitation spectrum from the emission at $2.41 \mathrm{eV}(515 \mathrm{~nm}$ ) (Figure 3B, green dashed) is broadened compared to 1 alone (Figure 3A, green dashed), as well as having a new feature described as a "fat" tail continuing towards higher energy. In 1 alone the emission at $2.74 \mathrm{eV}(453 \mathrm{~nm})$ dominantly comes from the FITC moiety but in the $100 \mu \mathrm{M}$ reaction of 1 seemingly everything contributes towards the emission. We believe this contributes to the broadening seen in the emission (Figure 2B). 
Emission from 1 alone has two assigned features: $2.40 \mathrm{eV}(516 \mathrm{~nm})$ from the FITC, and $2.92 \mathrm{eV}$ $(425 \mathrm{~nm}$ ) from CBT (Figure 2B and Figure S2). Upon activation emission from the FITC broadens from a FWHM of $0.21 \mathrm{eV}$ to at least $0.31 \mathrm{eV}$. A new feature emerges centered at $2.74 \mathrm{eV}(453 \mathrm{~nm})$ which is assigned as the emission from the resulting product. The lack of the $2.74 \mathrm{eV}(453 \mathrm{~nm})$ emission seen in 1 alone gives strong evidence for the identity (Figure 3A, yellow dotted).

Steady state emission intensity and anisotropy. With evidence that 1 exhibits spectral changes upon reduction-triggered aggregation, we turned to examine other emission characteristics of the compound that could be influenced by aggregation. To determine if these changes were due to reduction-triggered aggregation of $\mathbf{1}$, we also examined control compound $\mathbf{2}$, which lacks the disulfide bond necessary to trigger activation and aggregation in response to GSH. Aggregation of fluorescein is known to alter its spectral properties, including emission lifetime ${ }^{43}$ and quantum yield ${ }^{44}$, which reduces emission intensity. Fluorescein is also known to undergo homo-FRET (Fluorescence Resonance Energy Transfer, taking place between two identical fluorophore molecules), causing a depolarization of fluorescence emission from the sample ${ }^{43,45}$; this effect increases as more fluorophores come within FRET distance of each other ${ }^{45}$. We examined whether any of these trends were observed in $\mathbf{1}$ by measuring its steady-state emission intensity, anisotropy (polarization), and lifetime values at different concentrations, and comparing the results to that of $\mathbf{2}$ (Figure 4).

The emission intensity of both compounds was equal at $1 \mu \mathrm{M}$, while at higher concentrations a statistically significant reduction in emission intensity was measured for $\mathbf{1}$ when compared to $\mathbf{2}$ ( $p<$ 0.05), suggesting that triggered aggregation is reducing the quantum yield of FITC in $\mathbf{1}$ at these concentrations. At all concentrations, the anisotropy of $\mathbf{1}$ was lower than that of $\mathbf{2}$; as concentration increased, the anisotropy decreased for both compounds. The consistently lower anisotropy of $\mathbf{1}$ is attributed to aggregation of the oligomer products, reasonably bringing multiple FITC molecules within homo-FRET distance of each other. Models of aggregated CBT systems suggest that these aggregates 
pack together with an intermolecular distance of $0.37 \mathrm{~nm}^{46}$, which is well within the measured 5.3-5.7 $\mathrm{nm}$ Förster radius of $\mathrm{FITC}^{45}$. We attribute the concentration-dependent reduction of anisotropy possibly to low solubility for both compounds in this concentration range; indeed, general insolubility would result in compound packing to some extent and thus facilitate homo-FRET and a resultant reduction in anisotropy. While a distinct and significant $(p<0.05)$ difference in anisotropy is observed between $\mathbf{1}$ and the inactive control $\mathbf{2}$, the ideal probe should exhibit minimal self-depolarization before activation (while in the form of a single molecule, such as 2 ), but exhibit dramatic depolarization upon activation, attributed exclusively to the aggregation process. While this data indicates that aggregated $\mathbf{1}$ has consistently lower anisotropy than inactive small molecule $\mathbf{2}$, a cleaner, binary change in anisotropy is desired. Overall, the reduced emission intensity and anisotropy of $\mathbf{1}$ suggest that aggregation is indeed influencing the attached FITC fluorophore. We next investigated whether the aggregation process altered the emission lifetime of FITC to an extent that would enable lifetime-based imaging.

Time Resolved Emission Spectra. Time Resolved Emission Spectra (TRES) scans were performed to obtain the lifetimes of fluorescence emission. For $12 \mathrm{~nm}$ steps starting at $400 \mathrm{~nm}$ and ending at 700 $\mathrm{nm}$ an emission lifetime was collected from a $375 \mathrm{~nm}$ laser using TCSPC. From the lifetime map the corresponding time resolved emission can be reconstructed after correcting for the detector's response. The resulting TRES maps are shown in Figures S1, S3, and S4, and Table S1. Each lifetime from the TRES scan was fitted using the model shown in Table S1, using iterative reconvolution with the instrument response function (IRF), shown in Figure S1. Two chosen fits from the TRES map can be seen in Figures $\mathrm{S} 1(2.38 \mathrm{eV}, 520 \mathrm{~nm})$ and S3 $(3.01 \mathrm{eV}, 412 \mathrm{~nm})$. Using this method, we can measure lifetimes down to $\sim 200$ ps.

To better understand the dynamics, we constructed a Transient Emission Normalized Area Spectroscopy (TRANES) scan where each trace's area is normalized to unity ${ }^{47}$. This procedure reveals isoemissive points similar to isosbestic points in transient absorption spectroscopy (TAS). The resulting 
TRANES can be seen in Figure S5. First looking at the normal TRES for 1 alone, Figure S4a, the large peak at $\sim 2.8 \mathrm{eV}$, rising from $1.52 \mathrm{~ns}$ then decaying after $3.03 \mathrm{~ns}$ is assigned to the CBT emission. The peak at $2.4 \mathrm{eV}$ which appears at $1.52 \mathrm{~ns}$ reaching a maximum at $3.03 \mathrm{~ns}$ then decaying at $>18.24 \mathrm{~ns}$ is assigned to the FITC emission. Upon the reaction, Figure S4b, the CBT emission is now the prominent peak and a new emission appears filling the void at $2.7 \mathrm{eV}$. The new emission at $2.7 \mathrm{eV}$ is assigned to the resulting product material from the condensation. The FITC emission at $2.4 \mathrm{eV}$ is also broaden compared to the monomer but has similar decay. Comparing the TRES map and TRANES map, Figure S4b and S5b respectfully, there is now a clear isoemissive point at $2.45 \mathrm{eV}$. The emergence of the isoemissive point upon reaction is evidence of two emitting species, one being the product material from the condensation and the other, most likely being unreacted starting material. The trend continues in the reaction at $10 \mu \mathrm{M}$ where the isoemissive point is still present but not as clean as in the reaction at 100 $\mu \mathrm{M}$ and the decay line shape narrows with strong similarities to the monomer in both TRES and TRANES, (Figure S4c and S5c), thus giving more evidence there is both unreacted monomer and the product material in both concentrations of reactions.

Fluorescence emission lifetime at $2.38 \mathrm{eV}$ (532 nm) emission (FITC region) was $3.96 \mathrm{~ns}$ (100\%) for 1 only, consistent with the literature-reported lifetime of FITC small molecule ${ }^{48,49}$ (Figure S1). Upon reaction with GSH, the lifetime of 1 changed to $3.89 \mathrm{~ns}(71.37 \%)$ for the $1 \mu \mathrm{M}$ reaction mixture, $3.81 \mathrm{~ns}$ (78.91\%) for the $10 \mu \mathrm{M}$ reaction, and $3.87 \mathrm{~ns}(84.15 \%)$ for the $100 \mu \mathrm{M}$ reaction (Figure 4D, E). In comparison, the lifetime of 2 was $4.14 \mathrm{~ns}(67.47 \%)$ at $1 \mu \mathrm{M}, 4.06 \mathrm{~ns}(70.82 \%)$ at $10 \mu \mathrm{M}$, and $4.07 \mathrm{~ns}$ (70.20\%) at $100 \mu \mathrm{M}$ (Figure 4D, E). This major component is faster in $\mathbf{1}$ than in $\mathbf{2}$ at all concentrations tested, a trend also seen in the second, minor component of both compounds (Figure 4C). Overall, 1 mixed with a reducing agent exhibits a faster lifetime than both compound $\mathbf{2}$ and compound $\mathbf{1}$ in the absence of a reducing agent, suggesting that both the reduction-sensitive disulfide bond of $\mathbf{1}$ and the presence of a reducing agent are required to shorten the lifetime of the attached FITC. 
Aside from the FITC region, we also found a dramatic lifetime change at $3.01 \mathrm{eV} \mathrm{(412} \mathrm{nm,} \mathrm{CBT}$ region) (Figure S3, S4, and Table S1). The lifetimes from the fit are $0.32 \mathrm{~ns}(96.62 \%), 0.42 \mathrm{~ns}(95.81 \%)$, and 8.84 ns (98.04\%), for $\mathbf{1}$ alone, activated $\mathbf{1}$ at $10 \mu \mathrm{M}$, and activated $\mathbf{1}$ at $100 \mu \mathrm{M}$, respectively. This may suggest the packing of the aggregates after CBT condensation relies more on the CBT moiety, while the FITC moieties are arranged more toward the peripheral region. This observed dramatic lifetime change in the CBT moiety is difficult to apply to lifetime-based imaging because of significant cellular autofluorescence in the blue region of its emission profile (3.01 eV, $412 \mathrm{~nm})$.

Fluorescence Lifetime Imaging Microscopy of mammalian cells. Altered fluorescence lifetime has been used as a readout for many biochemical assays performed at the cellular level ${ }^{10}$. As 1 demonstrates reduction-triggered contrast in lifetime, we considered if this lifetime change could still be detected in the complex environment of the cell. First, we determined cellular uptake of the compounds by incubating HeLa cells with compound $\mathbf{1}$ (pre-activated by incubation with GSH), or with compound $\mathbf{1}$ or $\mathbf{2}$ alone. We examined the uptake of the compounds using fluorescence microscopy and flow cytometry (Figure 5). Fluorescence microscopy (Figure 5A) revealed notable differences in uptake patterns between the compounds: the pre-activated aggregates of 1 demonstrated high but diffuse uptake; compound $\mathbf{1}$ showed slightly higher intensity than $\mathbf{2}$ in HeLa cells, but $\mathbf{1}$ appears to be localized to particular areas within the cells, while $\mathbf{2}$ demonstrates the diffuse staining seen for pre-activated $\mathbf{1}$. The relative uptake in these samples was corroborated by flow cytometry results (Figure 5B, C).

To determine if the lifetime change of $\mathbf{1}$ could be detected in cells, HeLa cells were incubated with probes and lifetimes were measured using Fluorescence Lifetime Imaging Microscopy (FLIM, Figure 6A). The measured lifetimes for all three samples were best fit using bi-exponential functions (Figure 6B, D), in agreement with our previous lifetime measurements (Figure 4C-E). In cells, compound $\mathbf{1}$ preincubated with GSH exhibited shorter values for both lifetime components when compared to $\mathbf{2}$ or $\mathbf{1}$ added alone. This is also in agreement with the previous trend, where $\mathbf{1}$ exposed to a reducing agent 
reported faster lifetime components than $\mathbf{2}$. It is notable than in these cell samples, the faster of the two lifetime components $\left(\tau_{2}\right)$ became the major component for pre-activated $\mathbf{1}$ compared to $\mathbf{1}$ or $\mathbf{2}$ alone, resulting in a dramatic decrease ( $0.9 \mathrm{~ns})$ in the average lifetime of the reporter (Figure 6C, D). It is of note that when compound $\mathbf{1}$ was added to HeLa cells without addition of an exogenous reducing agent, no reduction in lifetime was observed in comparison with 2 . This may suggest that aggregation is not achieved to a suitable extent in this context, for example by condensation of the reduced CBT group onto thiol-containing biological molecules ${ }^{50-52}$ instead of another molecule of 1. Judging from the greater uptake of $\mathbf{1}$ into cells over $\mathbf{2}$ (Figure 5C), it is possible that $\mathbf{1}$ is activated (reduced) in cells but is interrupted before condensing with another molecule of $\mathbf{1}$, resulting in greater uptake and retention in cells when compared to $\mathbf{2}$, but without lifetime change that may come with aggregation. A more efficient self-assembly system may be achieved by tailoring the CBT scaffold reactivity ${ }^{37,42}$.

\section{Conclusions}

In conclusion, we report a reduction-sensitive fluorescent probe that exhibits an altered emission lifetime upon exposure to reducing conditions. A decrease in lifetime is observed when $\mathbf{1}$ is measured in the excitation and emission ranges of FITC, suggesting that the fluorophore is indeed influenced by the aggregation of the CBT scaffold. From this data, several improvements should be considered. In solution, notable lifetime changes are observed in the emission range of FITC only when compound $\mathbf{1}$ is incubated with a reducing agent such as GSH; the presence of a reduction-sensitive disulfide bond and a reducing agent seem to be required to facilitate the lifetime shift. However, it is important to discern the underlying mechanism(s) influencing the lifetime during aggregation: fluorophore aggregation can be associated with various quenching processes ${ }^{14,53}$, but in fluorescein and other reporters only some of these processes alter the emission lifetime ${ }^{43}$. With a better mechanistic understanding, the fluorophore and CBT scaffold could be tailored to direct these quenching processes and create a more dramatic 
lifetime change upon activation. While detected using FLIM, the lifetime decrease of $\mathbf{1}$ was not generated in situ in cells, suggesting that a careful selection of fluorophore and scaffold may also be required to allow the aggregation process to occur in the complex environment found within the cell. Along with this, the influence of concentration on the polarization and lifetime values leads us to believe a 'solubility switch' structure should be considered, in which the reduction reaction removes a highly water-soluble functional group, improving the solubility of the monomer while retaining the aggregation potential of the reduction product. Further study of this type of aggregation-induced lifetime contrast system can elucidate the mechanisms contributing to the observed lifetime change and illuminate synthetic design strategies to enhance the lifetime contrast produced by probe activation.

\section{Experimental Methods}

Chemical Synthesis. Experimental procedures and characterization data are provided in the Supplementary Information.

Steady state electronic absorption and emission, TRES. A solution of $\mathbf{1}(200 \mathrm{uM})$ in PBS (pH 7.4) was incubated with $\mathrm{GSH}(10 \mathrm{mM})$ and $\mathrm{NaHCO}_{3}(40 \mathrm{mM})$ and stirred for $30 \mathrm{~min}$ to $1 \mathrm{~h}$. Aliquots of the reaction mixture were then sequentially diluted using a $100 \mu \mathrm{M}$ dilution factor and a $10 \mu \mathrm{M}$ dilution factor. All solutions were in PBS buffer at pH 7.4. It is important to note spectra were measured in vitro as no purification or work up was done on the final reaction mixture before acquiring. Measuring in this manner best simulates a similar inhomogeneous mixture found in the triggered system. Steady state electronic absorption spectra were collected on a UV-2550 Shimadzu. Steady state emission, excitation, synchronous scan, and time resolved emission spectra (TRES) were collected on an Edinburgh Instruments FLS1000 Spectrometer. All spectra were collected with $1 \mathrm{~cm}$ path length quartz cuvette unless otherwise stated. Spectra was collected on a $1 \mu \mathrm{M}$ solution of 1 alone for comparison. 
Steady state fluorescence intensity and anisotropy, fluorescence lifetime. To 1 or $\mathbf{2}$ was added PBS buffer $(1 \mathrm{x}, \mathrm{pH}=7.4)$ to make $90 \%$ volume of the final solution at $1.11 \mathrm{x}$ the desired final concentration, then the solution was vortexed for $10 \mathrm{sec}$. A separate PBS solution of GSH (100 mM) and $\mathrm{NaHCO}_{3}(400 \mathrm{mM})$ was prepared, and the resulting solution was filtered with a 0.2 micron syringe filter. The filtered solution was diluted 10 -fold into the compound-PBS solution, to produce a final solution at $100 \%$ volume, containing $10 \mathrm{mM} \mathrm{GSH}, 40 \mathrm{mM} \mathrm{NaHCO}_{3}$, and a compound concentration of $100 \mu \mathrm{M}, 10$ $\mu \mathrm{M}$, or $1 \mu \mathrm{M}$. The solution was vortexed again for $10 \mathrm{sec}$ to $\mathrm{mix}$, then allowed to sit at room temperature for $30 \mathrm{~min}$ to 1 hour before measurements were collected. Fluorescence lifetime measurements were collected on an Edinburgh Instruments FLS1000 Spectrometer, excitation $488 \mathrm{~nm}$, emission $520 \mathrm{~nm}$. Samples for emission intensity and anisotropy were prepared similarly in a blackbottom 96-well plate for fluorescence measurements. Fluorescence intensity and anisotropy was measured on a SpectraMax M5 plate reader, with an excitation wavelength of $488 \mathrm{~nm}$, and an emission wavelength of $518 \mathrm{~nm}$.

Nanoparticle tracking analysis. To 1 or $\mathbf{2}$ was added PBS buffer ( $1 \mathrm{x}, \mathrm{pH}=7.4)$ to make $90 \%$ volume of the final solution at $1.11 x$ the desired final concentration, then the solution was vortexed for $10 \mathrm{sec}$. A separate PBS solution of $\mathrm{GSH}(100 \mathrm{mM})$ and $\mathrm{NaHCO}_{3}(400 \mathrm{mM})$ was prepared, and the resulting solution was filtered with a 0.2 micron syringe filter. The filtered solution was diluted 10 -fold into the compound-PBS solution, to produce a final solution at $100 \%$ volume, containing $10 \mathrm{mM} \mathrm{GSH}, 40 \mathrm{mM}$ $\mathrm{NaHCO}_{3}$, and a compound concentration of $100 \mu \mathrm{M}, 10 \mu \mathrm{M}$, or $1 \mu \mathrm{M}$. The solution was vortexed again for $10 \mathrm{sec}$ to $\mathrm{mix}$, then allowed to sit at room temperature for $30 \mathrm{~min}$ to 1 hour before measurements were collected. NTA was measured using a NanoSight NS300. To retain the correlation of size to compound concentration, the solutions were injected without further dilution. Sample flow rate was set to $50 \mathrm{\mu L} / \mathrm{min}$, and data was acquired as 5 replicates of 60 sec captures. Data was collected using a 
Blue488 laser, and all measurements were performed at $25 \mathrm{C}$ as maintained by the instrument. Particle size and particle concentration were calculated in accordance with the software default settings.

Cell culture and treatment. HeLa cells were cultured at 37$]^{\circ} \mathrm{C}$ in 10 ?] $\mathrm{cm}$ dishes containing Dulbecco's Modified Eagle's medium (DMEM) supplemented with 10\% fetal bovine serum and antibiotics (100] $\mathrm{U} / \mathrm{mL}$ penicillin, 100] $\mathrm{\mu g} / \mathrm{mL}$ streptomycin) under $5 \% \mathrm{CO}_{2}$ and $95 \%$ humidity.

Flow cytometry. HeLa cells were seeded in a 24 -well plate at a density of $3[\times] \times 10^{5}$ cells $/$ well (total of 12 wells) and cultured overnight. The next day, cells were incubated with pre-activated $\mathbf{1}(2 \mu \mathrm{M}$, 3 wells), 1 only ( $2 \mu \mathrm{M}, 3$ wells), 2 only ( $2 \mu \mathrm{M}, 3$ wells), or no probe ( 3 wells) for 1 hour, then washed with PBS buffer and digested with $0.25 \%$ trypsin. Cells were collected into Eppendorf tubes, washed with PBS buffer 3 times at 1250]x $\mathrm{g}$ for $3 \mathrm{~min}$, and resuspended in 200? $\mu \mathrm{L}$ PBS buffer. The fluorescence of cell samples (3 samples for each data point) was analyzed with a flow cytometer (Canto II , Becton Dickinson and Company, USA).

Fluorescence cell staining. HeLa cells were seeded on glass coverslips (0.13-0.16 mm thickness) at a density of $3 \times 10^{4}$ cells/well (total 4 wells) and cultured overnight. The next day, cells were incubated with pre-activated $\mathbf{1}(2 \mu \mathrm{M}, 1$ well), 1 alone ( $2 \mu \mathrm{M}, 1$ well), 2 alone ( $2 \mu \mathrm{M}, 1$ well) or no probe ( 1 well) for 1 hour, then fixed with $4 \%$ paraformaldehyde at $37{ }^{\circ} \mathrm{C}$ for $10 \mathrm{~min}$. The samples were washed with PBS 3 times, 5 min each. Cells were then incubated with DAPI for $5 \mathrm{~min}$ at room temperature. Coverslips were mounted on slides using ProLong Gold Antifade Mounting media and were used for fluorescence microscopy (Nikon Ti2, Japan) and fluorescence lifetime imaging microscopy described below.

Fluorescence lifetime imaging microscopy. Lifetime imaging was performed on a Leica TCS-SP8 microscope (Leica Microsystems GmbH, Wetzlar, Germany) with a PicoHarp 300 (PicoQuant, Berlin, Germany) lifetime module, possessing a resolution of $4 \mathrm{ps}$. Excitation was generated by a $488 \mathrm{~nm}$ white 
light laser with a $40 \mathrm{~Hz}$ pulse for 5-10 cycles. Emission was centered at $516 \mathrm{~nm}$ (range of 512 to $520 \mathrm{~nm}$ ). Images were processed with SymPhoTime 64 software (PicoQuant, Berlin, Germany). Results were processed with a multi-exponential (" $n$-exponential” function) tail fit, increasing the number of components until the resulting function provided a chi-squared value of approximately 1.0.

\section{Acknowledgements}

This work was supported by research grants to Prof. L. Cui from the University of New Mexico (UNM Startup Award), the National Institute of General Medical Sciences of National Institutes of Health (Maximizing Investigators' Research Award for Early Stage Investigators, R35GM124963), and the Career Development Award from the Department of Defense (W81XWH-17-1-0529). We are grateful to the support from the Department of Chemistry and Chemical Biology, University of New Mexico (UNM), the UNM Comprehensive Cancer Center and the National Cancer Institute of the United States (P30CA118100). NMR spectra were collected in part from the NMR Facility, Department of Chemistry and Chemical Biology, UNM, and from the Department of Medicinal Chemistry, College of Pharmacy, University of Florida (UF). Mass spectrometry services were provided in part by the Mass Spectrometry Facility, Department of Chemistry and Chemical Biology, UNM, and from the Mass Spectrometry Research and Education Center, Department of Chemistry, UF (NIH S10 OD021758-01A1). Nanoparticle Tracking Analysis instrumentation was provided by the UF Interdisciplinary Center for Biotechnology Research. Images in this paper were generated in the UNM Comprehensive Cancer Center Fluorescence Microscopy Shared Resource.

Keywords: fluorescence lifetime, fluorescein, cyanobenzothiazole, self-assembly

\section{Supporting Information.}

The Supporting Information is available on the bioRxiv preprint site. 


\section{References}

(1) Sarder, P., Maji, D., and Achilefu, S. (2015) Molecular probes for fluorescence lifetime imaging. Bioconjugate Chemistry 26, 963-974.

(2) Almutairi, A., Guillaudeu, S. J., Berezin, M. Y., Achilefu, S., and Frechet, J. M. (2008) Biodegradable $\mathrm{pH}$-sensing dendritic nanoprobes for near-infrared fluorescence lifetime and intensity imaging. Journal of the American Chemical Society 130, 444-445.

(3) Li, M., Ge, H., Arrowsmith, R. L., Mirabello, V., Botchway, S. W., Zhu, W., Pascu, S. I., and James, T. D. (2014) Ditopic boronic acid and imine-based naphthalimide fluorescence sensor for copper(II). Chem Commun (Camb) 50, 11806-9.

(4) Satapathy, R., Wu, Y. H., and Lin, H. C. (2012) Novel thieno-imidazole based probe for colorimetric detection of $\mathrm{Hg} 2+$ and fluorescence turn-on response of $\mathrm{Zn2+.} \mathrm{Org} \mathrm{Lett} \mathrm{14,} \mathrm{2564-7.}$

(5) Suhling, K., French, P. M., and Phillips, D. (2005) Time-resolved fluorescence microscopy. Photochem Photobiol Sci 4, 13-22.

(6) Berezin, M. Y., Kao, J., and Achilefu, S. (2009) pH-Dependent Optical Properties of Synthetic Fluorescent Imidazoles. Chemistry-a European Journal 15, 3560-3566.

(7) Okabe, K., Inada, N., Gota, C., Harada, Y., Funatsu, T., and Uchiyama, S. (2012) Intracellular temperature mapping with a fluorescent polymeric thermometer and fluorescence lifetime imaging microscopy. Nat Commun 3, 705.

(8) Kuimova, M. K., Yahioglu, G., Levitt, J. A., and Suhling, K. (2008) Molecular rotor measures viscosity of live cells via fluorescence lifetime imaging. J Am Chem Soc 130, 6672-3.

(9) Panda, D., Khatua, S., and Datta, A. (2007) Enhanced fluorescence of epicocconone in surfactant assemblies as a consequence of depth-dependent microviscosity. J Phys Chem B 111, 1648-56.

(10) Berezin, M. Y., and Achilefu, S. (2010) Fluorescence lifetime measurements and biological imaging. Chem Rev 110, 2641-84.

(11) Chib, R., Mummert, M., Bora, I., Laursen, B. W., Shah, S., Pendry, R., Gryczynski, I., Borejdo, J., Gryczynski, Z., and Fudala, R. (2016) Fluorescent biosensor for the detection of hyaluronidase: intensitybased ratiometric sensing and fluorescence lifetime-based sensing using a long lifetime azadioxatriangulenium (ADOTA) fluorophore. Anal Bioanal Chem 408, 3811-21.

(12) Lindgren, M., Sorgjerd, K., and Hammarstrom, P. (2005) Detection and characterization of aggregates, prefibrillar amyloidogenic oligomers, and protofibrils using fluorescence spectroscopy. Biophys J 88, 4200-12.

(13) Chen, N. T., Wu, C. Y., Chung, C. Y., Hwu, Y., Cheng, S. H., Mou, C. Y., and Lo, L. W. (2012) Probing the dynamics of doxorubicin-DNA intercalation during the initial activation of apoptosis by fluorescence lifetime imaging microscopy (FLIM). PLoS One 7, e44947.

(14) Swindlehurst, C. A., and Voss, E. W. (1991) Fluorescence measurements of immune complexes of Mab 4-4-20 with isomeric haptens. Biophysical Journal 59, 619-628.

(15) French, T., So, P. T. C., Weaver, J. D. J., Coelho-Sampaio, T., Gratton, E., Voss, J. E. W., and Carrero, J. (1997) Two-photon fluorescence lifetime imaging microscopy of macrophage-mediated antigen processing. Journal of Microscopy 185, 339-353.

(16) Esbjorner, E. K., Chan, F., Rees, E., Erdelyi, M., Luheshi, L. M., Bertoncini, C. W., Kaminski, C. F., Dobson, C. M., and Kaminski Schierle, G. S. (2014) Direct observations of amyloid beta self-assembly in live cells provide insights into differences in the kinetics of Abeta(1-40) and Abeta(1-42) aggregation. Chem Biol 21, 732-42.

(17) Boettcher, A., Gradoux, N., Lorthiois, E., Brandl, T., Orain, D., Schiering, N., Cumin, F., Woelcke, J., and Hassiepen, U. (2014) Fluorescence Lifetime-Based Competitive Binding Assays for Measuring the Binding Potency of Protease Inhibitors In Vitro. J Biomol Screen 19, 870-7. 
(18) Basuki, J. S., Duong, H. T., Macmillan, A., Erlich, R. B., Esser, L., Akerfeldt, M. C., Whan, R. M., Kavallaris, M., Boyer, C., and Davis, T. P. (2013) Using fluorescence lifetime imaging microscopy to monitor theranostic nanoparticle uptake and intracellular doxorubicin release. ACS Nano 7, 10175-89. (19) Garland, M., Yim, J. J., and Bogyo, M. (2016) A Bright Future for Precision Medicine: Advances in Fluorescent Chemical Probe Design and Their Clinical Application. Cell Chem Biol 23, 122-136.

(20) Ye, D., Shuhendler, A. J., Cui, L., Tong, L., Tee, S. S., Tikhomirov, G., Felsher, D. W., and Rao, J. (2014) Bioorthogonal cyclization-mediated in situ self-assembly of small-molecule probes for imaging caspase activity in vivo. Nat Chem 6, 519-26.

(21) Ye, D., Shuhendler, A. J., Pandit, P., Brewer, K. D., Tee, S. S., Cui, L., Tikhomirov, G., Rutt, B., and Rao, J. (2014) Caspase-responsive smart gadolinium-based contrast agent for magnetic resonance imaging of drug-induced apoptosis. Chemical Science 5, 3845-3852.

(22) Gomi, K., and Kajiyama, N. (2001) Oxyluciferin, a luminescence product of firefly luciferase, is enzymatically regenerated into luciferin. J Biol Chem 276, 36508-13.

(23) Liang, G., Ren, H., and Rao, J. (2010) A biocompatible condensation reaction for controlled assembly of nanostructures in living cells. Nat Chem 2, 54-60.

(24) Ren, H., Xiao, F., Zhan, K., Kim, Y. P., Xie, H., Xia, Z., and Rao, J. (2009) A biocompatible condensation reaction for the labeling of terminal cysteine residues on proteins. Angew Chem Int Ed Engl 48, 9658-62.

(25) Liu, X., and Liang, G. (2017) Dual aggregation-induced emission for enhanced fluorescence sensing of furin activity in vitro and in living cells. Chem Commun (Camb) 53, 1037-1040.

(26) Liang, G., Ronald, J., Chen, Y., Ye, D., Pandit, P., Ma, M. L., Rutt, B., and Rao, J. (2011) Controlled self-assembling of gadolinium nanoparticles as smart molecular magnetic resonance imaging contrast agents. Angew Chem Int Ed Engl 50, 6283-6.

(27) Ye, D., Pandit, P., Kempen, P., Lin, J., Xiong, L., Sinclair, R., Rutt, B., and Rao, J. (2014) Redoxtriggered self-assembly of gadolinium-based MRI probes for sensing reducing environment. Bioconjug Chem 25, 1526-36.

(28) Yuan, Y., Ge, S., Sun, H., Dong, X., Zhao, H., An, L., Zhang, J., Wang, J., Hu, B., and Liang, G. (2015) Intracellular Self-Assembly and Disassembly of (19)F Nanoparticles Confer Respective "Off" and "On" (19)F NMR/MRI Signals for Legumain Activity Detection in Zebrafish. ACS Nano 9, 5117-24.

(29) Yuan, Y., Ding, Z., Qian, J., Zhang, J., Xu, J., Dong, X., Han, T., Ge, S., Luo, Y., Wang, Y., et al. (2016) Casp3/7-Instructed Intracellular Aggregation of Fe3O4 Nanoparticles Enhances T2 MR Imaging of Tumor Apoptosis. Nano Lett 16, 2686-91.

(30) Miao, Q., Bai, X., Shen, Y., Mei, B., Gao, J., Li, L., and Liang, G. (2012) Intracellular self-assembly of nanoparticles for enhancing cell uptake. Chem Commun (Camb) 48, 9738-40.

(31) Dragulescu-Andrasi, A., Kothapalli, S. R., Tikhomirov, G. A., Rao, J., and Gambhir, S. S. (2013) Activatable oligomerizable imaging agents for photoacoustic imaging of furin-like activity in living subjects. J Am Chem Soc 135, 11015-22.

(32) Shen, B., Jeon, J., Palner, M., Ye, D., Shuhendler, A., Chin, F. T., and Rao, J. (2013) Positron emission tomography imaging of drug-induced tumor apoptosis with a caspase-triggered nanoaggregation probe. Angew Chem Int Ed Engl 52, 10511-4.

(33) Lin, J., Wang, W., Li, K., Huang, H., Lv, G., Peng, Y., Luo, S., and Qiu, L. (2017) Development of a kitlike radiofluorinated biomolecule leading to a controlled self-assembly of $18 \mathrm{~F}$ nanoparticles for a smart PET imaging application. Chem Commun (Camb) 53, 6476-6479.

(34) Ye, S., Wang, S., Gao, D., Li, K., Liu, Q., Feng, B., Qiu, L., and Lin, J. (2020) A New gammaGlutamyltranspeptidase-Based Intracellular Self-Assembly of Fluorine-18 Labeled Probe for Enhancing PET Imaging in Tumors. Bioconjug Chem. 
(35) Jiang, J., Zhao, Z., Hai, Z., Wang, H., and Liang, G. (2017) Intracellular Proteolytic Disassembly of Self-Quenched Near-Infrared Nanoparticles Turning Fluorescence on for Tumor-Targeted Imaging. Anal Chem 89, 9625-9628.

(36) Cao, C. Y., Chen, Y., Wu, F. Z., Deng, Y., and Liang, G. L. (2011) Caspase-3 controlled assembly of nanoparticles for fluorescence turn on. Chem Commun (Camb) 47, 10320-2.

(37) Ye, D., Liang, G., Ma, M. L., and Rao, J. (2011) Controlling intracellular macrocyclization for the imaging of protease activity. Angew Chem Int Ed Engl 50, 2275-9.

(38) Wang, Y., Li, J., Feng, L., Yu, J., Zhang, Y., Ye, D., and Chen, H. Y. (2016) Lysosome-Targeting Fluorogenic Probe for Cathepsin B Imaging in Living Cells. Anal Chem 88, 12403-12410.

(39) Gamcsik, M. P., Kasibhatla, M. S., Teeter, S. D., and Colvin, O. M. (2012) Glutathione levels in human tumors. Biomarkers 17, 671-91.

(40) Redmond, S. M. S., Joncourt, F., Buser, K., Ziemiecki, A., Altermatt, H.-J., Fey, M., Margison, G., and Cerny, T. (1991) Assessment of P-Glycoprotein, Glutathione-based Detoxifying Enzymes and O6Alkylguanine-DNA Alkyltransferase as Potential Indicators of Constitutive Drug Resistance in Human Colorectal Tumors.

(41) Ahmad, S., Okine, L., Le, B., Najarian, P., and Vistica, D. T. (1987) Elevation of glutathione in phenylalanine mustard-resistant murine $\mathrm{L} 1210$ leukemia cells.

(42) Chen, Z., Chen, M., Cheng, Y., Kowada, T., Xie, J., Zheng, X., and Rao, J. (2019) Exploring the Condensation Reaction between Aromatic Nitriles and Amino Thiols To Optimize In Situ Nanoparticle Formation for the Imaging of Proteases and Glycosidases in Cells. Angewandte Chemie-International Edition.

(43) Chen, R. F., and Knutson, J. R. (1988) Mechanism of fluorescence concentration quenching of carboxyfluorescein in liposomes: Energy transfer to nonfluorescent dimers. Analytical Biochemistry 172, 61-77.

(44) Arbeloa, I. L. (1981) Dimeric and trimeric states of the fluorescein dianion. Part 2.-Effects on fluorescence characteristics. J. Chem. Soc., Faraday Trans. 2 77, 1735-1742.

(45) Runnels, L. W., and Scarlata, S. F. (1995) Theory and application of fluorescence homotransfer to melittin oligomerization. Biophysical Journal 69, 1569-1583.

(46) Liu, S., Tang, A., Xie, M., Zhao, Y., Jiang, J., and Liang, G. (2015) Oligomeric hydrogels self-assembled from reduction-controlled condensation. Angew Chem Int Ed Engl 54, 3639-42.

(47) Periasamy, N. (2008) Heterogeneity of fluorescence determined by the method of area-normalized time-resolved emission spectroscopy. Methods in Enzymology 450, 21-35.

(48) Abbott, A. J., Amler, E., and Ball, W. J., Jr. (1991) Immunochemical and spectroscopic characterization of two fluorescein 5'-isothiocyanate labeling sites on $\mathrm{Na}+\mathrm{K}(+)$-ATPase. Biochemistry 30 , 1692-701.

(49) Hungerford, G., Benesch, J., Mano, J. F., and Reis, R. L. (2006) Effect of the labelling ratio on the photophysics of fluorescein isothiocyanate (FITC) conjugated to bovine serum albumin.

(50) Ramil, C. P., An, P., Yu, Z., and Lin, Q. (2016) Sequence-Specific 2-Cyanobenzothiazole Ligation. J Am Chem Soc 138, 5499-502.

(51) Zheng, Z., Chen, P., Li, G., Zhu, Y., Shi, Z., Luo, Y., Zhao, C., Fu, Z., Cui, X., Ji, C., et al. (2017) Mechanistic study of CBT-Cys click reaction and its application for identifying bioactive $\mathrm{N}$-terminal cysteine peptides in amniotic fluid. Chem. Sci. 8, 214-222.

(52) Miao, Q. Q., Li, Q., Yuan, Q. P., Li, L. L., Hai, Z. J., Liu, S., and Liang, G. L. (2015) Discriminative Fluorescence Sensing of Biothiols in Vitro and in Living Cells. Analytical Chemistry 87, 3460-3466.

(53) Chen, H., Ahsan, S. S., Santiago-Berrios, M. B., Abruna, H. D., and Webb, W. W. (2010) Mechanisms of quenching of Alexa fluorophores by natural amino acids. J Am Chem Soc 132, 7244-5. 
A
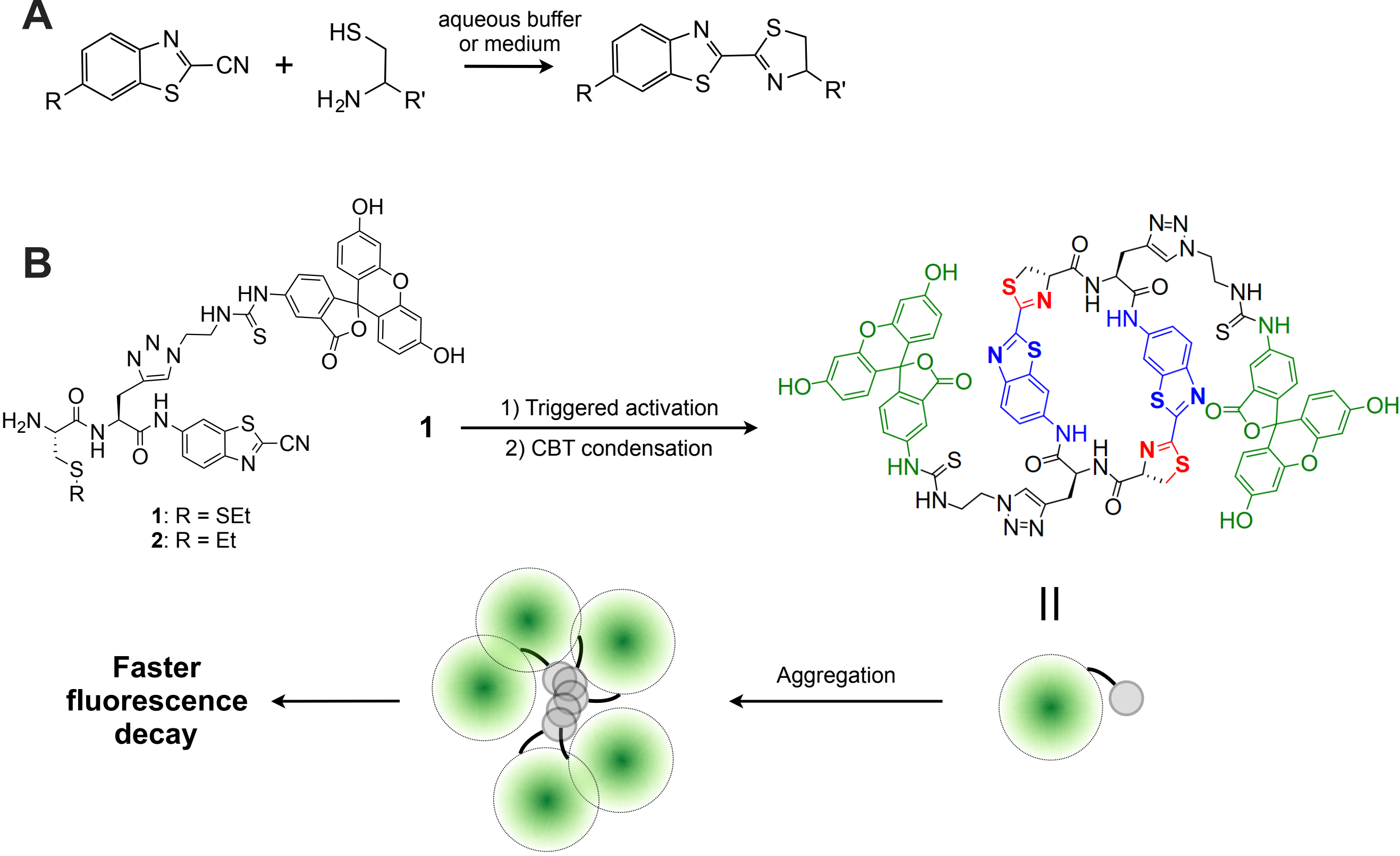

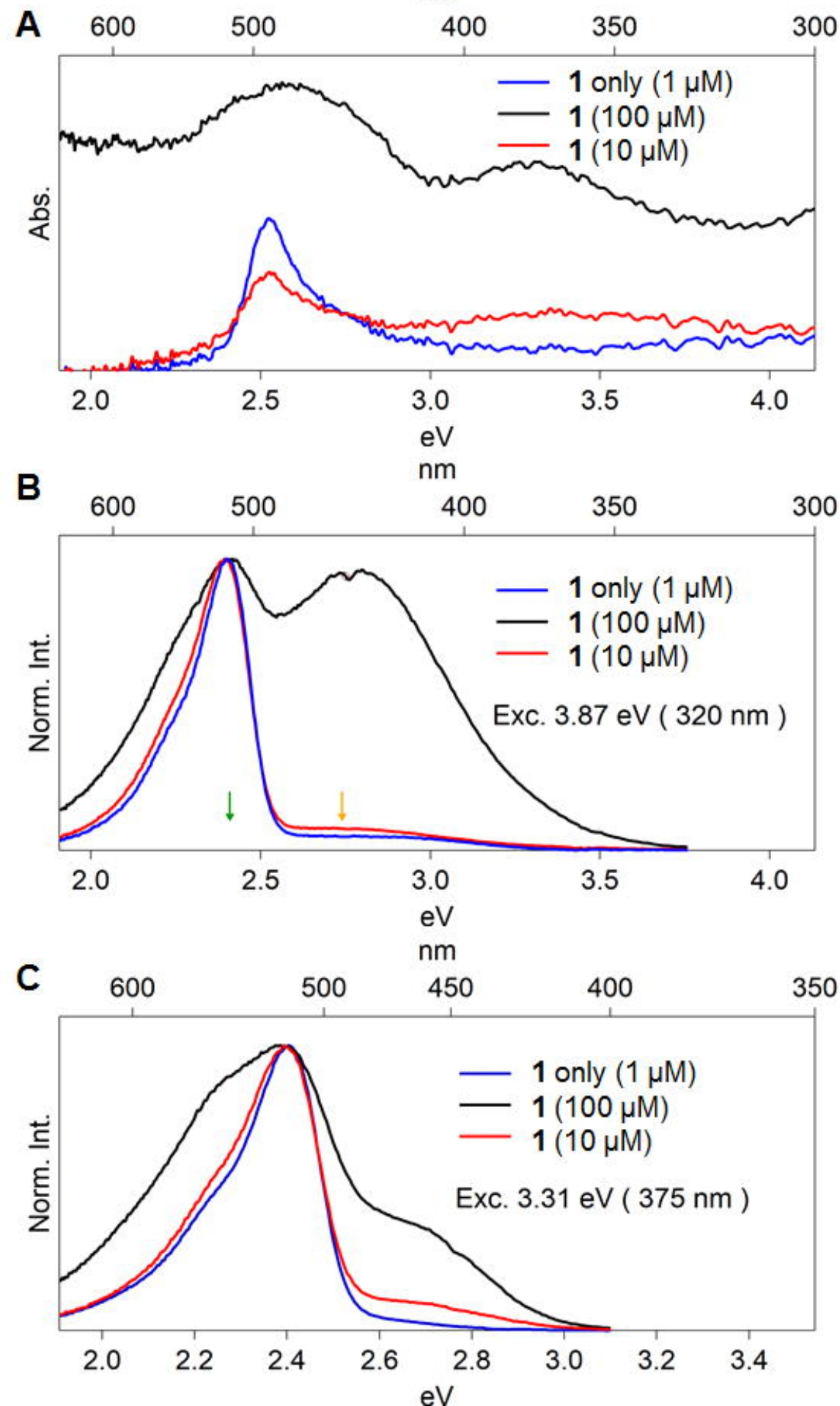
A $\mathrm{nm}$

$\begin{array}{llllll}6000 & 500 & 400 & 350 & 300\end{array}$
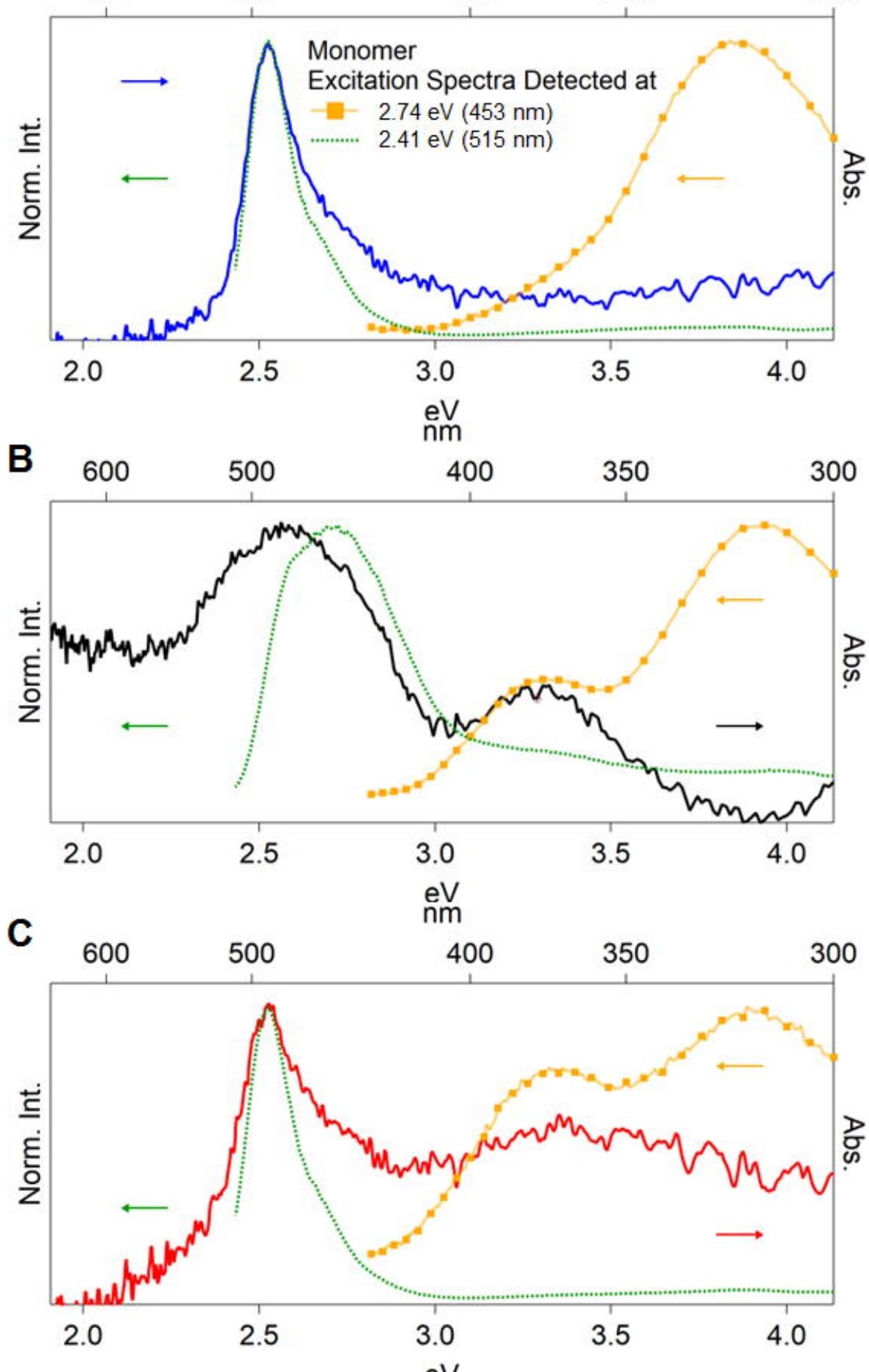


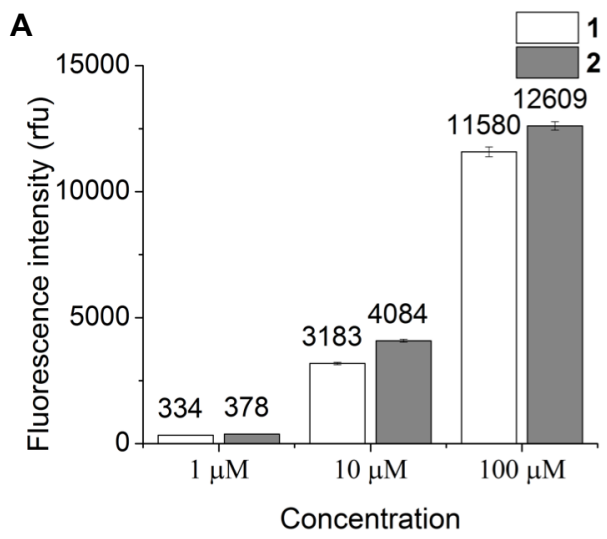

C

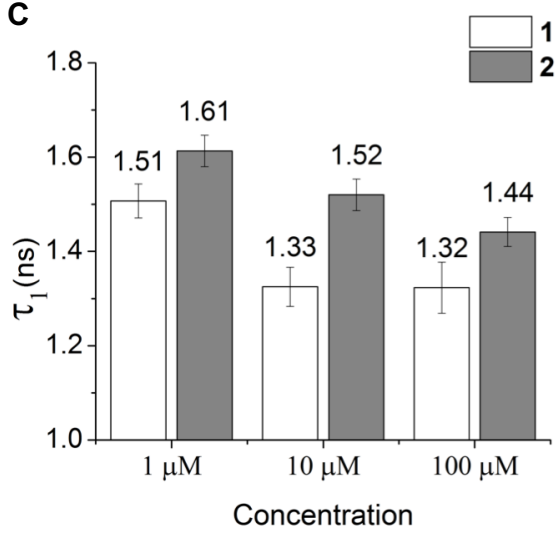

D
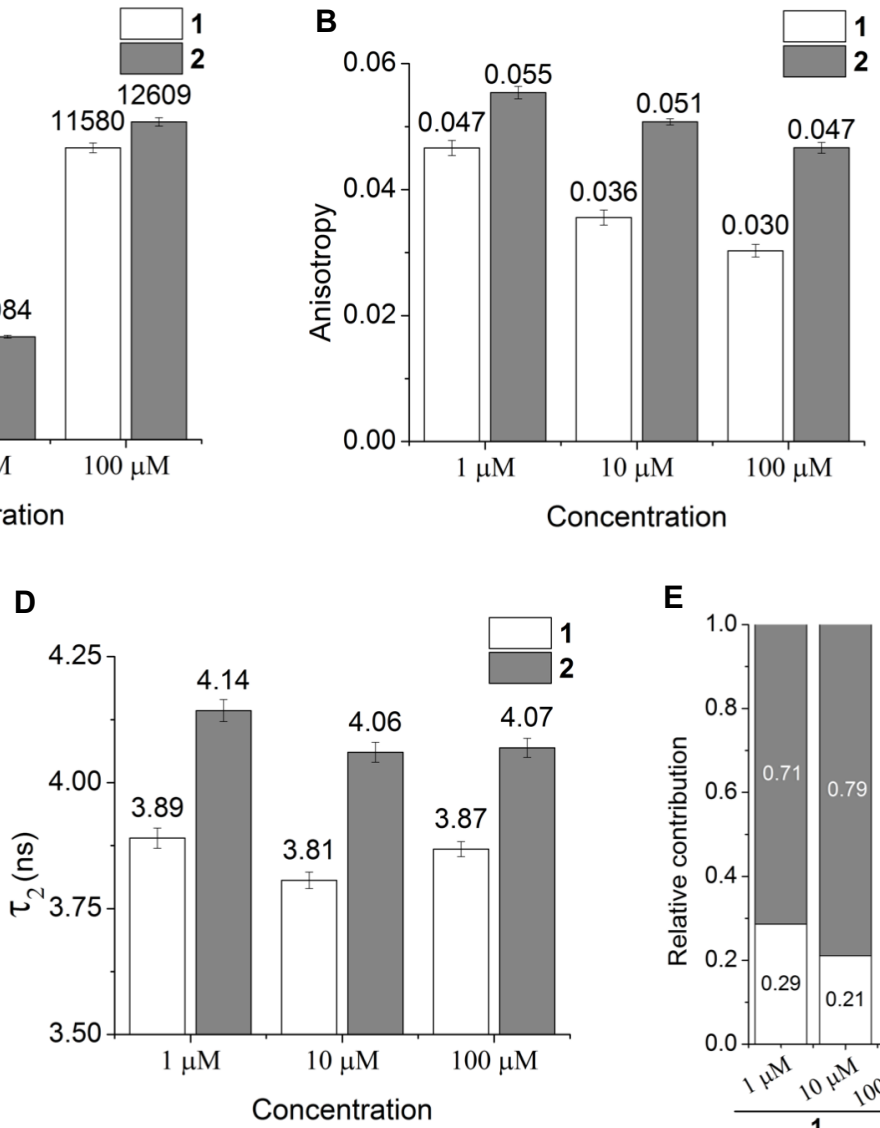

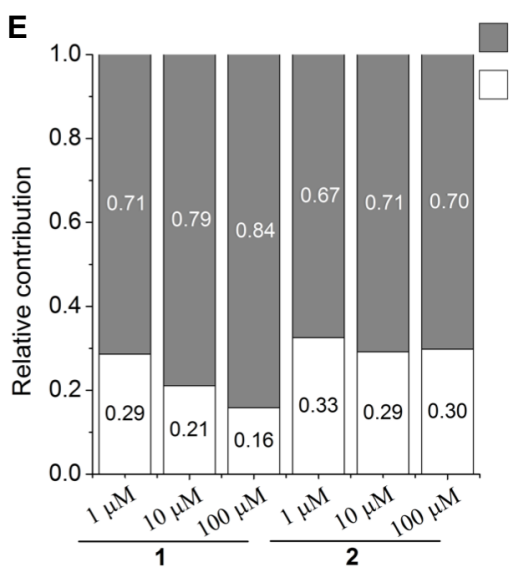




\section{cell only}
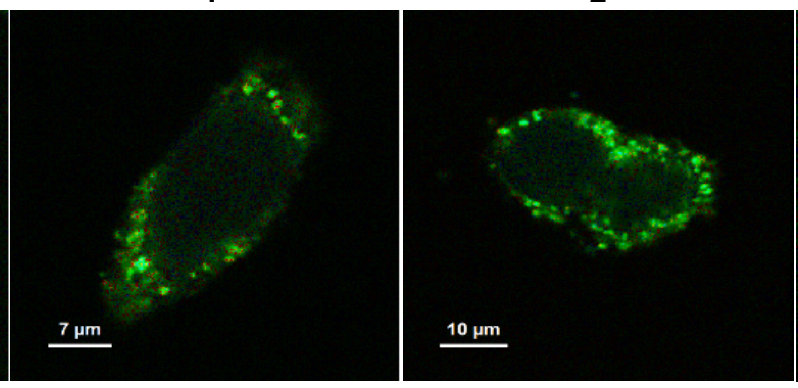

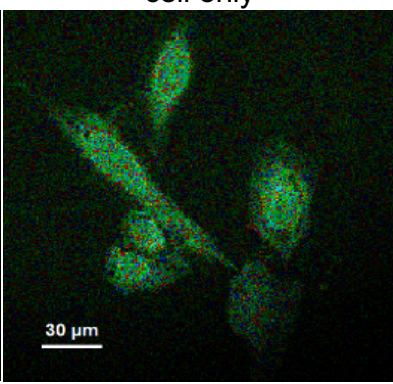

-

\section{C}

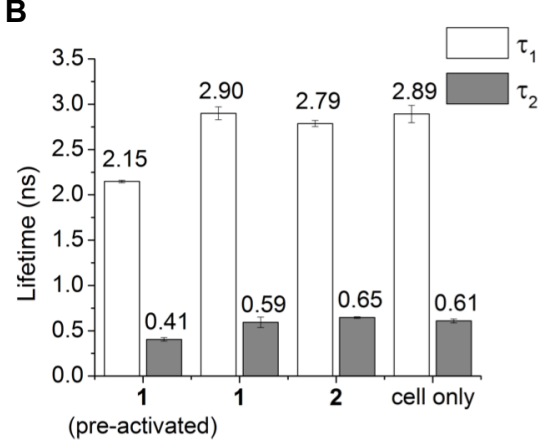

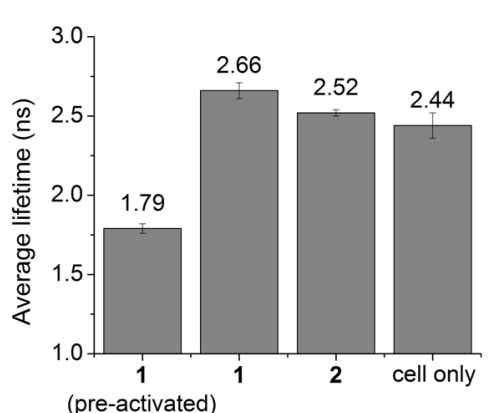

\section{D}

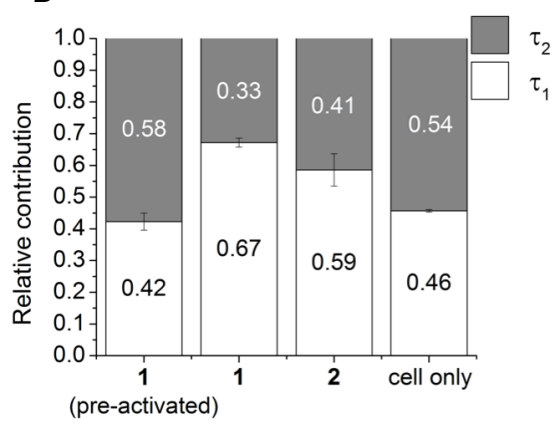

